Cahiers d'études italiennes

23 | 2016

FILIgRANA

Intuizione e forma. André Jolles: vita, opere, posterità

\title{
Il necrologio come «forma semplice» nella letteratura contemporanea
}

Obituary As a "Simple Form" of Contemporary Literature

Andrea Rondini

\section{(2) OpenEdition}

\section{Journals}

Edizione digitale

URL: http://journals.openedition.org/cei/3250

DOI: 10.4000/cei.3250

ISSN: 2260-779X

\section{Editore}

UGA Éditions/Université Grenoble Alpes

\section{Edizione cartacea}

Data di pubblicazione: 30 décembre 2016

Paginazione: $211-222$

ISBN: 978-2-84310-337-7

ISSN: 1770-9571

Notizia bibliografica digitale

Andrea Rondini, «ll necrologio come «forma semplice» nella letteratura contemporanea», Cahiers d'études italiennes [Online], 23 | 2016, online dal 23 janvier 2017, consultato il 26 mars 2021. URL: http://journals.openedition.org/cei/3250 ; DOI: https://doi.org/10.4000/cei.3250 


\title{
IL NECROLOGIO COME «FORMA SEMPLICE» NELLA LETTERATURA CONTEMPORANEA
}

\author{
Andrea Rondini \\ Università di Macerata
}

Le «forme semplici» di André Jolles costituiscono un momento fondamentale della teoria letteraria novecentesca. Esse possiedono in prima istanza una spendibilità in riferimento alle testualità più direttamente previste dal modello teorico di partenza. Tuttavia la ricezione della proposta del critico olandese può anche esplicarsi da un lato nel ritrovamento di «forme semplici attualizzate» in opere contemporanee, e dall'altro nel tentativo di costruire alcune "nuove forme semplici» utili a individuare modi specifici della comunicazione letteraria.

Per un'esemplificazione di una linea di continuità delle forme jollesiane si consideri la presenza del Caso ${ }^{\mathrm{I}}$ ne I sommersi e $i$ salvati di Primo Levi, in cui esso si configura come porzione di testo riconoscibile, pressoché dotata di autonomia. Si presenta qui brevemente il Caso (ma il testo leviano ne espone ulteriori, ad esempio quello rappresentato da Chaim Rumkovski ${ }^{2}$ ) di una ragazza di sedici anni, stipata in una camera a gas insieme ad altri deportati, che viene ritrovata miracolosamente viva da una Squadra Speciale, un Sonderkommando, il gruppo di prigionieri addetti a svuotare le camere dai cadaveri e prepararle per i successivi massacri (i Sonderkommandos stessi costituiscono a loro volta un Caso ${ }^{3}$ ). Ecco il racconto di Levi, che tra l'altro connota la vicenda proprio in termini di Caso:

Viene chiamato un medico, che rianima la ragazza con una iniezione [...]. In quel momento sopraggiunge Mushfeld, uno dei militi SS addetti agli impianti di morte; il medico lo chiama da parte e gli espone il caso. Mushfeld esita, poi decide: no, la

I. A. Jolles, Forme semplici, in Id., I travestimenti della letteratura, S. Contarini (a cura di), Milano, Bruno Mondadori, 2003, pp. 379-399.

2. P. Levi, I sommersi e i salvati, in Opere, II, M. Belpoliti (a cura di), Torino, Einaudi, 1997, pp. I038-I044.

3. Ivi, pp. $1028 \mathrm{sgg}$. 
ragazza deve morire; se fosse più anziana il caso sarebbe diverso, avrebbe più senno, forse la si potrebbe convincere a tacere su quanto le è accaduto, ma ha solo sedici anni: di lei non ci si può fidare. Tuttavia non la uccide di mano sua, chiama un suo sottoposto che la sopprime con un colpo alla nuca ${ }^{4}$.

Sul versante invece delle «forme semplici di nuova generazione» si veda l'esplicito riferimento a Jolles in recenti studi sulla presenza della diceria e del pettegolezzo nei testi letterari a partire dall'Eneides.

Piace in proposito sottolineare come la teoria letteraria contemporanea tenda a concepire le "forme semplici» in chiave dinamica, cercando di alleggerirne la natura archetipale ${ }^{6}$. Del resto già Jolles aveva posto una correlazione tra la Leggenda e il moderno record sportivo (in questo ripreso successivamente da Jauss, anch'egli convinto di tale possibile attualizzazione $)^{7}$.

Si propone in questa sede — senza avere la minima pretesa di gareggiare con il paradigma originario, ma solo per provarne la vitalità — di verificare l'esistenza di una «forma semplice» collegata alla rappresentazione della morte e associabile in particolare a un riuso letterario del necrologio. Si può seguire la linea tracciata dallo stesso Jolles quando, analizzando il Caso, ne conia dicitura ed esistenza ${ }^{8}$.

La morte è stata resa discorso attraverso molteplici forme enunciative come l'epitafio, la laudatio funebris, l'exitus illustrium virorum o l'ars bene moriendi. Occorrerà subito sottolineare che l'epitafio presentava una funzione pubblica e sociale allo stesso modo in cui la "forma semplice» - per esempio la Saga — veniva narrata «durante le occasioni solenni o comunque importanti» ${ }^{9}$. Il discorso necrologico si muove quindi tra letteratura e funzione extraletteraria e per questo può essere affiancato alle "forme semplici», che si collocano, almeno in prima battuta, in una zona preletteraria della scrittura. Occorre naturalmente aggiungere che per il Novecento, oltre ai generi classici sopra citati, risulta decisivo il modello

4. Ivi, p. I034. (Corsivi nostri.)

5. S. Sini, Di bocca in bocca: pettegolezzi, dicerie, calunnie nella rappresentazione letteraria, "Mantichora", I, dicembre 20II, <http://ww2.unime.it/mantichora/wp-content/uploads/2OI2/oI/Mantichora-I-pag-7OI-7I9Sini.pdf> [consultato il 4 luglio 2016].

6. N. Pasero, Le forme semplici e la cultura popolare: alcune considerazioni, in S. M. Barillari (a cura di), Motti, arguzie, facezie e altre "forme semplici» della cultura popolare, Roma, Meltemi, 200o, p. 23.

7. A. Jolles, Forme semplici, cit., pp. 299-300; H. R. Jauss, Alterità e modernità nella letteratura medievale, trad. di M. G. Saibene Andreotti e R. Venuti, presentazione di C. Segre, Torino, Bollati Boringhieri, 1989 [Alterität und Modernität der mittelalterlichen Literatur, in Gasammelte Aufsätze 1956-1976, München, Fink, 1977], p. 43. In particolare, Jolles parla dei campioni dell'atletica leggera, Jauss dei campioni di calcio.

8. A. Jolles, Forme semplici, cit., pp. 379-380.

9. Ivi, p. 304 . 
letterario necrologico per eccellenza, vale a dire la Spoon River Anthology pubblicata nel I9Is da Edgar Lee Masters ${ }^{10}$.

Verranno qui presi in considerazione, soprattutto da un punto di vista morfologico, quei testi letterari dalla misura tendenzialmente breve ${ }^{\mathrm{II}}$ in cui la componente mortuaria è dominante, e che condividono con la forma preletteraria del necrologio la caratteristica della concisione, la propensione variabile alla riduzione della letterarietà, il presentarsi in forma autonoma (assoluta o inserita in narrazioni più ampie), la possibilità di collegarsi ad altri necrologi, i cenni alla biografia del defunto. Si tratta naturalmente di elementi non sempre compresenti in un'unica realizzazione testuale, ma che contraddistinguono la funzione necrologica. In questo senso si può pensare di stabilire, pur nella diversità, un collegamento in particolare con il vero e proprio topos letterario dell'exitus illustrium virorum, che trova nella morte di Seneca narrata da Tacito ${ }^{\mathrm{I} 2}$ uno dei suoi momenti più alti ${ }^{13}$. Si cercherà infine di mostrare come il necrologio, dal punto di vista della sintassi del racconto, non sia solo presenza testuale «statica» ma possa assumere un più marcato aspetto diegetico.

Il necrologio letterario può venire concepito come testo indipendente o essere inserito in una struttura narrativa più ampia, allo stesso modo in cui un Caso - lo si è già visto con Levi — può essere rinvenuto in un saggio oppure ancora un aforisma ${ }^{14} \mathrm{o}$ un Memorabile ${ }^{\mathrm{IS}}$ possono essere incastonati in un romanzo. Le forme necrologiche sono inoltre spesso intrecciate al genere della biografia breve o microbiografia. Già Jolles aveva rilevato

Io. E. L. Masters, Spoon River Anthology, New York, Macmillan, I9I5.

II. Sulle forme brevi si veda M. Curcio (a cura di), Le forme della brevità, Milano, Franco Angeli, 20I4. Già Zumthor aveva guardato a Jolles nelle sue riflessioni sulle forme brevi: P. Zumthor, La brièveté comme forme, in M. Picone, G. Di Stefano, P. D. Stewart (a cura di), Genèse, codification et rayonnement d'un genre médiéval : le nouvelle, actes du colloque international de Montréal (McGill University, I4-I6 octobre I982), Montréal, Plato Academic Press, "Bibliotheca Romanica», 1983, pp. 3-8.

I2. Tacito, Annali, C. Questa, B. Ceva (a cura di), Milano, Rizzoli, I990, vol. II, XV, 62-63, pp. 753-755.

13. A. Ronconi, Exitus illustrium virorum, in A. L. Trombetti Budriesi (a cura di), Un gallo ad Asclepio. Morte, morti e società tra antichità e prima età moderna, Bologna, Clueb, 2013, pp. 389-406.

I4. U. Eco, Note sull'aforisma. Statuto aletico e poetico del detto breve, in G. Ruozzi (a cura di), Teoria e storia dell'aforisma, Milano, Bruno Mondadori, 2004, p. I52 ( Una prima distinzione è quella tra aforismi creati ex professo e intenzionalmente brevi e aforismi per estrazione, la cui brevità nasce da un atto di amputazione da contesto più vasto. Ho visto che anche André Jolles in Forme semplici distingue fra citazioni e apoftegmi. Ma Jolles è il primo ad ammettere che, se pure massima e proverbio nascono sempre per atto individuale, dal momento che il popolo o la tradizione se ne impadroniscono essi diventano quel che sono, brevi testi che circolano avulsi da ogni contesto - e non potremmo dire che una citazione non abbia tutte le buone qualità di un aforisma solo per il fatto che nasce da un contesto più ampio. Quello che conta è la sua proverbialità, icasticità, capacità di convincere, indipendentemente dal quadro da cui proviene».)

15. G. Savelli, L'ambiguità necessaria. Zeno e il suo lettore, Milano, Franco Angeli, 1998, p. 66. Ogni capitolo della Coscienza di Zeno di Svevo contiene una serie di episodi, "storielle», per esempio il racconto delle ultime sigarette o quello dello schiaffo del padre, che sono assimilabili a quello "che Jolles ha definito come il Memorabile». 
l'importanza della biografia per alcune «forme semplici», mostrando come la Leggenda si basasse sulle Vite dei Santi' ${ }^{16}$ (tra le quali la Legenda aurea di Jacopo da Varazze), nelle quali il Santo, la cui esistenza spesso si concludeva con il martirio, diveniva figura da imitare ${ }^{17}$. Il confine tra narrazione della morte e biografia è metodologicamente rilevante. Soprattutto nel caso di volumi composti da microbiografie - genere a sua volta dotato di una propria fisionomia ${ }^{18}$ - esse tendono non poche volte verso il necrologio e contengono spesso, come zona specifica, il racconto degli ultimi istanti di vita ${ }^{\text {I9 }}$. Particolarmente interessanti in questo senso si rivelano le considerazioni di Dino Baldi sul rapporto tra descrizione della morte e genere biografico antico, nel quale il trapasso assumeva posizione fortemente simbolica perché coronava l'idea di esistenza come paradigma ${ }^{20}$.

Le premesse teoriche fin qui svolte possono essere verificate sui testi. Un vero e proprio necrologio rintracciabile nel tessuto della diegesi narrativa è il paragrafo che nella Tregua di Primo Levi viene dedicato a Hurbinek:

Hurbinek, che aveva tre anni e forse era nato in Auschwitz e non aveva mai visto un albero; Hurbinek, che aveva combattuto come un uomo, fino all'ultimo respiro, per conquistare l'entrata nel mondo degli uomini, da cui una potenza bestiale lo aveva bandito; Hurbinek, il senza nome, il cui minuscolo avambraccio era pure stato segnato col tatuaggio di Auschwitz; Hurbinek morì ai primi giorni del marzo I945, libero ma non redento. Nulla resta di lui: egli testimonia attraverso queste mie parole $^{21}$.

Si trova qui un esempio paradigmatico di una delle funzioni principali della «forma semplice» necrologica: quella testimoniale, volta a costruire il ricordo di una Vittima (attivo in nuce anche nell'episodio della sopravvissuta nella camera a gas). Una funzione che si basa su una strategia retorico-onomastica, legata alla ripetizione del nome Hurbinek.

Una variante di questo modello è rintracciabile in un racconto di Winfried Georg Sebald che nasce come ampliamento di un necrologio letto su un giornale: l'incipit stesso del racconto è a sua volta un necrologio, in una sorta di dialogo metanarrativo tra discorso letterario e «forma semplice» di partenza:

Nel gennaio del 1984 mi giunse da S. la notizia che Paul Bereyter, di cui ero stato allievo alle elementari, aveva messo fine ai suoi giorni la sera del 30 dicembre, esattamente

I6. A. Jolles, Forme semplici, cit., pp. 273-274 e sgg.

I7. Ivi, pp. 282-283.

I8. Si veda per esempio il recente G. Alvi, Eccentrici, Milano, Adelphi, 20I5.

19. D. Baldi, Vite efferate di papi, Macerata, Quodlibet, 2015.

20. D. Baldi, Morti favolose degli antichi, Macerata, Quodlibet, 20Io, p. II.

2I. P. Levi, La tregua, in Id., Opere, I, M. Belpoliti (a cura di), Torino, Einaudi, 1997, p. 2 I6. 
una settimana dopo aver compiuto settantaquattro anni [...]. Il necrologio dal titolo Lutto per un amato concittadino, pubblicato nell' "Allgäuer Anzeigeblatt» e inviatomi insieme con la notizia, non diceva che Paul Bereyter si era tolto la vita - per libera scelta o in preda a un raptus autodistruttivo - , ma parlava soltanto dei meriti del pedagogo defunto, delle cure che aveva dedicato ai suoi scolari, ben oltre il proprio dovere, della sua passione per la musica, della sua ricchezza di idee e così via. A dire il vero, nel necrologio, si osservava anche, ma solo di sfuggita, che il Terzo Reich aveva interdetto Paul Bereyter dall'esercizio della sua professione di insegnante. Questa frase, alquanto incongrua e incidentale, così come la risolutezza con cui Paul Bereyter si era dato la morte mi indussero negli anni successivi a riflettere sempre più spesso su di lui, e a tentare infine [...] una ricognizione della sua storia, a me di fatto sconosciuta ${ }^{22}$.

Le pagine sebaldiane seguenti al ritratto funebre iniziale — anche qui scandito dalla ripetizione ossessiva del nome - si configurano dunque come una biografia-necrologio, una biografia che completa un necrologio e ne è la versione articolata e ampliata.

L'inserzione del ricordo funebre sembra ormai in qualche modo una tecnica acquisita nella narrativa contemporanea, per lo meno in quelle poetiche che intendono attraversare gli snodi dolorosi della Storia passata e presente; si considerino, solo per fare un esempio, i necrologi di alcuni caduti nella Prima e nella Seconda Guerra Mondiale inseriti da Eraldo Affinati come controcanto delle vicende del giovane immigrato africano in Vita di vita ${ }^{23}$. Ed è forse lecito chiedersi se non possa anche essere richiamata, per tutte le opere con vocazione necrologica, una categoria sulla quale alcuni studiosi di letteratura contemporanea hanno richiamato l'attenzione, vale a dire quella di pietas ${ }^{24}$.

$\mathrm{Vi}$ sono testi in cui la sezione necrologica tende a staccarsi in modo più netto e ad assumere nel contempo tassonomia catalogatoria. In Trieste di Daša Drndić (che si presenta come "romanzo documentario» e quindi in una zona liminale rispetto alla pura fictio) viene descritta la vita a Trieste negli anni dell'occupazione nazista. La narratrice, Haya Tedeschi, dopo aver attraversato quegli anni, redige un dossier dei nazisti attivi nel capoluogo giuliano, in particolare presso la Risiera di San Sabba. Il dossier - di cui si danno qui due esempi — assume questa forma:

Paul Bredow, SS-Unterscharführer. Nato nel 1902. Infermiere. In servizio a: Grafeneck, Hartheim, Sobibór, Treblinka, Trieste. Hobby: sparare su bersagli mobili. Media

22. W. G. Sebald, Paul Bereyter, in Id., Gli emigrati, trad. di A. Vigliani, Milano, Adelphi, 2007 [Die Ausgewanderten, Frankfurt am Main, Eichborn, 1992], pp. 37-38.

23. E. Affinati, Vita di vita, Milano, Mondadori, 2014.

24. R. Palumbo Mosca, "Tutto torna alla notte tranne questa grande parola. Diciamo: d'amore». La pietas nel romanzo, primi approcci, "Narrazioni», 4, 20I4, pp. 93-IO3. 
raggiunta a Sobibór: cinquanta ebrei al giorno. Un debole per i profumi. Dopo la guerra, abbandona San Sabba insieme al collega Karl Frenzel e lavora come falegname a Giessen. Muore nel 1945 in un incidente stradale a Göttingen ${ }^{25}$.

Erich Bauer, SS-Oberscharführer, nato nel 1900. Di bassa statura ma di solida costituzione, è un uomo particolarmente crudele. Dirige il lavoro delle camere a gas a Sobibór. Viene dislocato in Italia, a San Sabba, dal I943 al 1945. È catturato nel 1950, quando, in un luna park di Berlino, viene riconosciuto da un ex prigioniero sopravvissuto, tale Samuel Lerer. Nel I95I viene condannato a morte e dopo l'abolizione della pena di morte in Germania la condanna è commutata all'ergastolo. Muore in carcere a Berlino nel $198 \mathrm{O}^{26}$.

Per la forma paratattica che quasi rinuncia a elevarsi dal grado zero della scrittura, tale necrologio si pone in una zona pressoché extraletteraria; non a caso Trieste arriva a un limite per cui non solo la letterarietà, ma addirittura la scrittura, si ritira fino a lasciare solo il nome, in una radicalizzazione della traccia onomastica: il romanzo ospita infatti un elenco di 9000 nomi e cognomi di «ebrei deportati dall'Italia, oppure uccisi in Italia e nei Paesi che l'Italia ha occupato tra il 1943 e il $1945^{{ }^{27}}$ e che si sviluppa per ben 52 pagine del libro ${ }^{28}$, ognuna divisa in quattro colonne di nomi. Si riporta uno stralcio dell'elenco:

\author{
Tedeschi Ada \\ Tedeschi Adelaide \\ Tedeschi Adele \\ Tedeschi Adolfo \\ Tedeschi Alberto Sebastiano \\ Tedeschi Arrigo \\ Tedeschi Bonaventura detta Ines \\ Tedeschi Bianca \\ Tedeschi Bice \\ Tedeschi Emanuele Amedeo \\ Tedeschi Emma \\ Tedeschi Emma Bianca \\ Tedeschi Ermenegilda \\ Tedeschi Ernesta Irma
}

L'elenco funebre delle vittime si duplica quando un ex-allievo di Haya le invia una lettera che contiene i nomi degli appartenenti alla propria famiglia, la famiglia Piazza:

25. D. Drndić, Trieste, trad. di L. Avirović, Milano, Bompiani, 2015 [Sonnenschein, Zaprešić, 2007], pp. $252-253$.

26. Ivi, p. 248.

27. Ivi, p. 166.

28. Ivi, pp. I67-219. 
Piazza Alceo, Piazza Angelo, Piazza Antelo, Piazza Anita, Piazza Bruno, Piazza Donato, Piazza Edvige, Piazza Elio, Piazza Elisa, Piazza Elvira, Piazza Emanuele, Piazza Fernanda, Piazza Giacomo, Piazza Gina, Piazza Gino, Piazza Giuseppe, Piazza Maria Luisa, Piazza Rachele, Piazza Regina, Piazza Sed Angelo, Piazza Sed Camilla, Piazza Sed Cesira, Piazza Sed Consola, Piazza Sed Costanza, Piazza Sed Emma, Piazza Sed Ester, Piazza Sed Eugenio, Piazza Sed Leda, Piazza Sed Marco, Piazza Sed Rosa, Piazza Sed Sara, Piazza Umberto, Piazza Virginia ${ }^{29}$.

È questa la punta di massima coincidenza con la «forma semplice necrologica» e il punto di massima lontananza dall'exitus. Si tocca qui anche un elemento di contatto con la Saga: essa «in epoche di crisi funzionerebbe da coagulante attraverso l'attivarsi di principî quali la consanguineità e il vincolo di parentela, la vendetta del sangue e la faida, il concubinaggio e la proscrizione extra-familiare, l'ereditarietà e il sostituirsi di un ramo collaterale a un ramo legittimo. Tale straordinaria ipotesi, lasciata da André Jolles a uno stato germinale, avrebbe il vantaggio di spiegare» il fiorire di cicli etnici: «ad esempio, solo dopo la chiusura dei ghetti la letteratura ebraica produce una lunga serie di romanzi genealogico-familiari ${ }^{30}$. Lidea di Calabrese apre la strada allo stesso necrologio, forma narrativa inseribile in testualità genealogiche.

Dotata di icastica autonomia, sebbene inserita nella fluviale narrazione, è pure La parte dei delitti in 2666 di Roberto Bolaño. Si tratta di una sezione corposa del romanzo, strutturata in sequenze cadenzate di paragrafi, anche visivamente staccati, in cui si descrivono il ritrovamento di IIo donne (anche ragazzine) assassinate a Santa Teresa (Messico), una breve sintesi della loro misera vita e le modalità del loro omicidio. Ogni paragrafo consta in media di due o tre pagine e può comprendere anche la narrazione degli istanti o dei giorni precedenti la morte. Quasi sempre sono indicati nome, età, professione, luogo del ritrovamento, tipologia di colpo mortale ricevuto. Il necrologio, che in prima istanza possiede una natura eminentemente descrittiva, recupera una sua valenza diegetica: entità in sé conchiusa, la forma necrologica non di rado si dispone a costruire una catena, un catalogo collegato alle dinamiche narrative. Si legga il necrologio, tra i più brevi e compatti, di Marta Navales Gómez, una delle donne dell'immenso femminicidio, metafora della mostruosità della Storia:

A ottobre, nella discarica del complesso industriale Arsenio Farrell, fu ritrovata una nuova vittima. Si chiamava Marta Navales Gómez, vent'anni, un metro e settanta

29. Ivi, p. I3I.

30. S. Calabrese, www.letteratura.global, Torino, Einaudi, 2005, p. I83. 
di altezza, capelli castani, lunghi. Erano due giorni che mancava da casa. Indossava una vestaglia e dei fuseaux che i suoi genitori non riconobbero come suoi. Era stata violentata ripetute volte per via anale e vaginale. La morte era ascrivibile a strangolamento. La cosa curiosa è che Marta Navales Gómez lavorava alla Aiwo, una maquiladora giapponese del complesso industriale El Progreso. Eppure il suo corpo era stato ritrovato nel complesso industriale Arsenio Farrell, nella discarica, un posto complicato da raggiungere in macchina, a meno che la macchina non fosse un mezzo della spazzatura. Fu scoperta da alcuni bambini, di mattina, e dopo mezzogiorno, quando fu rimosso il cadavere, un folto gruppo di operai si avvicinò all'ambulanza per vedere se si trattava di un'amica, di qualche compagna o di una semplice conoscente ${ }^{31}$.

Si potrebbe proseguire: «nel novembre I994 fu rinvenuto in un appezzamento di terreno incolto il cadavere mezzo bruciato di Silvana Pérez Arjona. Aveva quindici anni ed era magra, di carnagione scura, un metro e sessanta di altezza. I capelli neri le arrivavano fin sotto le spalle, anche se quando fu ritrovato il cadavere erano per metà strinati» ${ }^{32}$.

Bolaño è abituato a muoversi lungo il confine tra epitafio e biografia, come dimostrano anche le microbiografie de La letteratura nazista in America $^{33}$ (delle quali sia consentito almeno citare en passant quella di Daniela De Montecristo, vera summa della poetica dello scrittore cileno ${ }^{34}$ ).

Siamo all'interno di vere e proprie "enciclopedie di morti», per ricordare il titolo di un famoso libro di Danilo Kiš, non estraneo alla modellistica letteraria qui presentata ${ }^{35}$. Ci si può chiedere anche se le donne di Bolaño (ma anche Hurbinek o Bereyter) rappresentino una variante dei martiri, di santi laici: i nuovi personaggi illustri sono le Vittime (del resto è già stato messo in luce il rapporto tra vita, agiografia ed exitus illustrium virorum $)^{36}$.

La caratteristica tassonomica si accentua decisamente in Mosche d'inverno di Eugenio Baroncelli, che descrive, come recita il sottotitolo, "27I morti in due o tre pose» ${ }^{37}$. Si tratta di un archivio formato da microbiografie concentrate in una pagina (a volte mezza) di personaggi più o meno famosi (anche

3I. R. Bolaño, 2666, trad. di I. Carmignani, Milano, Adelphi, 2009 (2007-2008) [2666, Barcelona, Ignacio Echevarrìa, 2004], p. 425. La maquiladora è una fabbrica straniera di assemblaggio.

32. Ivi, pp. 462-463.

33. R. Bolaño, La letteratura nazista in America, trad. di M. Nicola, Milano, Adelphi, 2013 [La literatura nazi en América, Barcelona, Seix Barral, 1996].

34. Ivi, pp. 99-Ioo.

35. D. Kiš, Enciclopedia dei morti, trad. di L. Costantini, Milano, Adelphi, 1996 (I988) [Enciklopedija mrtvih, Zagreb, Beograd, Globus, Prosveta, 1983].

36. F. Fonio, Dalla legenda alla novella: continuità di moduli e variazioni di genere. Il caso di Boccaccio, "Cahiers d'études italiennes", 6, 2007, pp. I27-I8I.

37. E. Baroncelli, Mosche d'inverno. 27 morti in due o tre pose, Palermo, Sellerio, 2010. 
di finzione: Madame Bovary) la cui morte è suddivisa in alcune sezioni: Cari agli dei; Cuori infranti; Di cosa?; Di freddo; Di gioia; Di spada; Di un male; Fantasmi; Folli; Fumatori; Insonni; Per acqua; Per caso e non per caso; Per fuoco; Per scelta; Vecchi. Con il libro di Baroncelli si evidenziano, e per certi versi si confermano, due caratteristiche fondamentali della «forma semplice necrologica». La prima è la riduzione dell'autorialità: Baroncelli ha aggiunto di suo solo in minima parte ("Quasi niente di questo libro è mio. Le epigrafi sono state dettate da altri [...]; i fatti [...] appartengono a chi li ha vissuti [...]. A me il lettore può addebitare senz'altro quel poco che resta», vale a dire "una data sbagliata per imprevidenza o un tramonto scrupolosamente inventato, qualche aggettivo triste e due o tre avverbi malinconici» $\left.{ }^{3}\right)$. Inoltre, Mosche d'inverno si struttura come catalogo: la morfologia catalogatoria, la "vertigine della lista» ${ }^{39}$, è anch'essa attributo primario del paradigma della semplicità in quanto rimanda a quella riduzione del caos tematizzata da Jolles ${ }^{40}$. Una struttura rafforzata dai richiami interni alle varie morti, visibili ad esempio per Arthur Koestler e Walter Benjamin ${ }^{4}$.

Tali micronarrazioni - spesso introdotte da un proverbio - ripropongono la variante d'en bas del paradigma della morte illustre. Riportiamo due esempi, Hemingway (dalla sezione Per scelta) e Trakl (dalla sezione Cari agli dei):

\section{Ernest Hemingway}

Ketchum, Idaho, 2 luglio 196r. All'alba, gettato quel che resta del suo Avana, con l'amorosa cura di un orefice appoggia le canne del fucile al centro della fronte e fa fuoco. Sveltisce il tempo, che si trascina come un elefante. Insegue quel destino da trentatré anni, da quando Clarence, fragile padre, si è puntato l'arma alla tempia e ha fatto fuoco ${ }^{42}$.

\section{Georg Trakl}

Ospedale di Cracovia, 3 novembre I9I4. Il cuore gli si ferma nel miserando padiglione psichiatrico in cui è stato ricoverato. Ha abusato dell'oppio nei bordelli di Salisburgo, del cloroformio nella farmacia in cui è stato praticante e della sua vita quando era vivo. Solo la poesia non l'ha tradito, ma questo non lo sa. Gli mancano due mesi per compiere ventotto anni, quanti ne ha l'obnubilata Edie Sedgwick quando finisce come lui. Gli mancano tre anni per sapere che sua sorella Greta, che lo ha baciato

38. Ivi, p. II.

39. U. Eco, Vertigine della lista, Milano, Bompiani, [2009] 2012.

40. A. Jolles, Forme semplici, cit., pp. 271-272. Si veda anche A. Inglese, Il romanzo e la strategia dellinventario, «Nazione Indiana», 27 marzo 2013: <https://www.nazioneindiana.com/2013/03/27/gli-ideali-della-letteratura-moderna-e-la-strategia-dellinventario/> [consultato il I6 luglio 2016].

4I. E. Baroncelli, Mosche d'inverno, cit., pp. I9I e 196.

42. Ivi, p. 195 . 
per la prima volta nel buio, mentre ascoltavano il primo coro del Tristano, si ucciderà con la pistola. Nessuno lo accompagna alla tomba, tranne quel devoto minatore di Hallstadt che gli faceva da attendente ${ }^{43}$.

La forma-catalogo presiede alla struttura anche delle Morti favolose degli antichi di Baldi ${ }^{44}$. L'autore rielabora in modo creativo le fonti consultate, dedicate a personaggi storicamente esistiti ${ }^{45}$ (con l'eccezione di Ulisse). Morti favolose degli antichi è suddiviso in rubriche: Morti di poeti; Morti di atleti e pensatori; Morti di re, condottieri, tiranni e imperatori; Morti di popoli, città ed eserciti; Celesti sparizioni; Selvaggi omicidi; Suicidi controvoglia; Morti per mano dei parenti; Morti improvvise per cause naturali; Suicidi a testa alta; Quasi morti, quasi vivi.

Baldi sottolinea la concezione costruttiva e «vitale» della morte presso gli antichi, coronamento fisico, ma anche semioticamente denso, dell'esistenza $^{46}$, un modo ancora una volta - Jolles docet - di conferire ordine al momento estremo e per certi versi più significativo della vita.

Ecco la morte (per suicidio) di Arria:

Arria era la moglie di Cecina Peto. Il marito era malato, di un male senza scampo. Anche il figlio era malato, e anch'egli mortalmente: un figlio bellissimo e a loro carissimo, più per tutte le sue buone qualità che per essere nato da loro. Un giorno morì, e Arria ne predispose il funerale senza far saper nulla al marito, per non peggiorare il suo stato. Con lui fingeva che il figlio fosse vivo e anzi stesse meglio; alle sue domande rispondeva in modo appropriato: «Ha riposato, mangia bene». Nei momenti in cui il dolore traboccava, usciva dalla camera e si abbandonava alle lacrime; poi si asciugava gli occhi, si ricomponeva, rasserenava il volto e tornava al capezzale del malato, lasciando, per così dire il lutto fuori dalla porta. Quando le parve che non ci fosse più motivo per andare oltre, prese un pugnale, se lo immerse nel petto, lo estrasse e lo porse al marito, dicendo: «Credimi, Peto, non fa male» ${ }^{47}$.

Morti favolose degli antichi sembra quindi possedere un senso morale ${ }^{48}$; tuttavia, non tutte le morti possono essere ascritte a tale perimetro, come sembra evidenziare il brevissimo capitolo dedicato a Pasicle di Efeso:

Pasicle fu arconte di Efeso. Una notte, all'uscita da un banchetto, venne assalito da alcuni suoi nemici, e scappò. Gli inseguitori tuttavia erano in difficoltà, a causa del buio. Quando furono nei pressi del tempio di Era, la madre di Pasicle, che era lì

\footnotetext{
43. Ivi, pp. 28-29.

44. D. Baldi, Morti favolose degli antichi, cit.

45. D. Baldi, Nota ai testi, ivi, pp. I7-I8.

46. D. Baldi, Premessa, ivi, pp. II-I6.

47. Ivi, pp. 325-326.

48. Ibid.
} 
sacerdotessa, sentendo dei rumori prese una lampada e uscì fuori. In questo modo senza volerlo svelò agli assalitori dove fosse il figlio, e lo fece uccidere ${ }^{49}$.

Con Tumbas di Cees Nooteboom il necrologio assume una spiccata o comunque più evidente apertura diegetica, perché in questo caso il dispositivo necrologico si ibrida con la narrazione della visita alla tomba. Si riporta integralmente - per dare compiuta idea della sua fisionomia e come conclusione della piccola mappa qui proposta - il capitolo dedicato a Herman Melville, uno degli ottanta scrittori e poeti presso il cui sepolcro Nooteboom si reca in pellegrinaggio:

La cartina del Woodlawn Cemetery è verde come il mare, i vialetti che lo attraversano sono serpentine bianche in tutto quel verde, circoscrivono spazi dove sono sepolti i morti e che hanno nomi come Holly, Hemlock, Wintergreen e Sassafras. Sono arrivato fin qui con la Lexington Avenue Subway, una linea che normalmente si prende solo per andare da una stazione all'altra di Manhattan e che in genere è sovraffollata. Ora corre attraverso l'isola, vola lungo il Bronx, I6Ist Street, I76th Street, I83rd Street. Via via che salgono i numeri, spariscono i bianchi. Le stazioni hanno ora nomi veri e propri, Fordham Road, Moshulu Parkway, a parte noi sono tutti neri, finché anche gli ultimi passeggeri scendono e restiamo soli a sfrecciare verso il XIX secolo. Scendiamo in quella che sembra una terra di nessuno, l'unico essere umano è una ragazza ferma a una stazione dell'autobus che ci indica la strada per Woodlawn. La città appare all'improvviso lontanissima. Entriamo dall'ingresso in 233rd Street. Woodlawn è un nome adatto, tutto sembra subito campestre, dolci declivi, alberi alti e antichi, mausolei con colonnati greci, qui giaceva o giace la crème di una città ricca. La tomba di Melville è semplice, quasi povera, la sepoltura di un autore dimenticato al momento della morte. In questi giorni di patriottismo qualcuno ci ha messo sopra una bandierina americana. Hart Crane ha scritto sulla sua visita qui una poesia di cui non vengo del tutto a capo. Parla di dadi fatti con le ossa degli annegati che ci lasciano un messaggio; relitti che ci scorrono davanti senza il suono della campana di bordo - parole meste scritte nel linguaggio della marineria; parla di bussola, quadrante e sestante e, alla fine, di un uomo la cui ombra è custodita solo dal mare. Ciò che risuona in questi versi è un doppio dolore: non solo quello per la morte dello scrittore, ma anche l'amarezza per l'altra morte, quella della sua opera finché lui era in vita. Melville si era imbarcato a ventidue anni sull'Acushnet, poi aveva abbandonato la nave alle Isole Marchesi, dove era rimasto per qualche tempo. Su quei viaggi aveva scritto i suoi primi libri e aveva avuto successo, ma le cose erano cambiate con Moby Dick: disprezzo, derisione dei critici, oblio, il manoscritto di Billy Budd mai pubblicato in vita, un altro romanzo perduto per sempre, i tour di conferenze per mettere insieme qualche soldo, un lavoretto alla dogana di New York. Del successo postumo non saprà mai nulla, un'umiliante solitudine.

Piccoli astri candidi, una quercia maestosa, vento che strappa i fiori bianchi della magnolia e li fa cadere come una strana specie di neve, il figlio trentacinquenne 
morto dieci anni prima di lui, sui due lati della lapide edere di pietra e, lontanissimo, il rumore della città dove, in ogni biblioteca e in ogni libreria, si trovano i suoi libri ${ }^{\text {so }}$.

La fenomenologia necrologica appare quindi ricca di modulazioni interne; per esempio, anche laddove si rimanga fedeli alla grammatica della concisione può tuttavia variare lo statuto della voce enunciativa: Franco Arminio compone una serie di microtesti che si potrebbero definire autonecrologi, fulminee descrizioni che i defunti fanno del proprio trapasso, tra ironia e amari bilanci esistenziali: «Avevo appena finito di vedere la televisione. Mi sentivo debole. Mi sono disteso sul divano e ho sentito come una mano gigantesca che mi premeva il cuore. Ho pensato che stavo morendo e non avevo comprato il loculo. Sicuramente mi avrebbero messo sotto terra. E questo era l'ultimo fallimento della mia vita» ${ }^{5 \mathrm{I}}$. Oppure, su una linea paradossale e sarcastica: «Sono morto alle sette del mattino. Un modo come un altro per cominciare la giornata ${ }^{52} . » \mathrm{E}$ anche: «Io stavo a Zurigo. Sul manifesto hanno scritto che sono salita alla casa del padre. La verità è che mi sono buttata dal quinto piano»" ${ }^{53}$.

Ma il grande discorso di Jolles non si esaurisce in una serie di pur fondamentali paradigmi formali, nella grammatica delle istituzioni letterarie, perché apre orizzonti gnoseologici. Sembra allora che il nostro sia il tempo dell'Archivio e della Vittima ${ }^{54}$ (e del suo Carnefice), il tempo della Memoria e della Morte catalogata. Non è detto che tutti siano d'accordo con tale risultanza, che meriterebbe ampia trattazione specifica. In ogni caso, senza le capacità euristiche delle "forme semplici», il dibattito non potrebbe nemmeno iniziare.

50. C. Nooteboom, Tumbas. Tombe di poeti e pensatori, trad. di F. Ferrari, Milano, Iperborea, 2015 [Tumbas. Gräber von Dichtern und Denkern, München, Schirmer \& Mosel, 2006], pp. 233-234.

5I. F. Arminio, Cartoline dai morti, Roma, Nottetempo, 20I0, p. 9.

52. Ivi, p. 86.

53. Ivi, p. 93.

54. Per una disamina, anche polemica, verso questo paradigma, D. Giglioli, Critica della vittima. Un esperimento con l'etica, Roma, Nottetempo, 2014. 